\title{
$1 \quad$ Motor effort and adaptive sampling in perceptual decision-making
}

2

3

4

5 Tianyao $\mathrm{Zhu}^{1 *}$

6

7

8

$9 \quad{ }^{1}$ Faculty of Education and Psychology, Eötvös Loránd University, Budapest, Hungary

10

11

12

$13 *$ Corresponding author

14 E-mail: tianyao.a.zhu@ gmail.com (TZ)

15 


\section{Abstract}

People usually switch their attention between the options when trying to make a decision. In our experiments, we bound motor effort to such switching behavior during a twoalternative perceptual decision-making task and recorded the sampling patterns by computer mouse cursor tracking. We found that the time and motor cost to make the decision positively correlated with the number of switches between the stimuli and increased with the difficulty of the task. Specifically, the first and last sampled items were chosen in an attempt to minimize the overall motor effort during the task and were manipulable by biasing the relevant motor cost. Moreover, we observed the last-sampling bias that the last sampled item was more likely to be chosen by the subjects. We listed all possible Bayesian Network models for different hypotheses regarding the causal relationship behind the last-sampling bias, and only the model assuming bidirectional dependency between attention and decision successfully predicted the empirical results. Meanwhile, denying that the current decision variable can feedback into the attention switching patterns during sampling, the conventional attentional drift-diffusion model (aDDM) was inadequate to explain the size of the lastsampling bias in our experimental conditions. We concluded that the sampling behavior during perceptual decision-making actively adapted to the motor effort in the specific task settings, as well as the temporary decision.

\section{Introduction}

6 When people try to choose between two similar products in a shopping center, they often approach each shelf where the products are displayed to have a closer look. If the choice is difficult to make, people may walk back-and-forth the two shelves for a long time. Many 
people will start by examining the product around the entrance of the shop, but choose the one

40 near the checkout counter eventually to save some effort. This daily example suggests that our

decisions are not solely shaped by the relative values of the alternatives, but also other factors including the motor effort related to the sampling and the action execution processes. until recently. It is still an on-going controversy whether action is part of decision-making: According to the Embodied Choice model, action execution is part of the decision-making process rather than merely a means to report the decision; in other words, action can feedback into the decision-making process [1]. Researchers have also studied decision-making by analyzing movement patterns [2] and sought neural imaging evidence for the involvement of the sensorimotor system during decision-making [3]. the movement toward one of the options, as the Embodied Choice model proposed, but rather the difference in the required motor effort during action. Other studies also reported the influence of motor effort during action upon decision-making: For example, perceptual decisions have been observed to be biased by the difference in the motor cost to make the response [5]. Moreover, the exposure to the unequal motor cost also biased the subsequent decisions even when they were vocally reported, indicating that motor effort can affect decision-making at a stage earlier than action execution [6]. De Lange and Fritsche [7] suggested that motor cost can influence decision-making similarly to rewards. Besides, motor effort can also affect changes of mind during decision-making [8].

Apart from action, the sampling behavior can be accompanied by motor effort as well, especially when the items to choose from are spatially separated. However, no investigation has focused on the influence of motor effort upon sampling. Although in some paradigms two or more visual stimuli were present, the main form of movement involved during sampling 
64 was the saccadic eye movement; unlike limb movements, energy costs are not a significant

65 consideration in the planning for saccades [9].

66 Another issue following the separation of the options in space is the attention

67 allocation during sampling. Typically, the decision-maker switches the attention (by the

68 behavior of switching the gaze) between the options at least once, sometimes multiple times.

69 What is the relationship between attention and decision-making? Several results showed that

70 manipulation of attention biased the decision [10-14]. Under the assumption that attention can

71 influence value integration during decision-making, Krajbich et al. [15] proposed the

72 attentional drift-diffusion model (aDDM). Unlike the traditional drift-diffusion model where

73 the relevant evidence accumulates at a constant rate (the drift rate) within one decision, the

74 aDDM allows the drift rate to change with attention: the option currently being attended

75 (gazed at) shall receive more evidence. Such a model has successfully explained the gaze

76 patterns and several gaze-related biases in preferential and perceptual decisions performed by

77 human subjects [15-17].

Specifically, the aDDM assumes that attention or gaze switches between the options

79 randomly. In fact, there is rare evidence supporting that temporary choices can influence

attention allocation. Shimojo et al. [18] reported the gaze cascade effect that gaze was biased

toward the finally chosen item during preferential decision-making, yet Krajbich [19] argued

that the phenomenon was readily explained by the aDDM and suggested that gaze or attention has a causal effect on choice, but not vice versa.

Under natural circumstances, humans gather information and sample relevant cues with attention and active sensing behaviors (shift of gaze and assisting limb/body movement) [20]. Sampling behavior itself can be regarded as a low-level decision-making process about what information to acquire, as well as where and when [21]. In the current study, we aim to 
89

90

91

92

93

94

95

96 Methods

5

\section{Paradigm and stimuli}

8

task, especially how sampling behavior adapts to the expected motor effort given the specific environment of the task. We designed a paradigm in which motor effort was bind to the sampling and action execution processes, and manipulated the expected motor cost to examine corresponding changes in the sampling patterns. Additionally, we tested the causal relationship between the temporary decision and the attention allocation strategy during sampling by analyzing a Bayesian Network model and simulating an aDDM.

The paradigm was based on a two-alternative perceptual decision-making task in which subjects were asked to decide which of the two groups of black and white dots contained more white ones. Two imaginary circles (diameter $3.5 \mathrm{~cm}$ ) were located horizontally apart on the upper half of the screen $(20 \mathrm{~cm}$ between their centers), each containing 100 dots. The dots were either black or white on a $50 \%$ gray background. In each trial, we randomly set the proportions of white dots in each group with the following method: First, we separately drew an average proportion $A$ from $[0.4,0.6]$ and a distinction proportion $D$ from $[0,0.3]$. The proportions of white dots in the two groups would be $A \pm 0.5 D$. Then, we randomly assigned the two calculated proportions to the left and right group, making sure that in $50 \%$ trials there were more white dots in the left group.

To bind motor effort to the sampling process, we applied an artificial rule that the sampling quality is in proportion to the distance between the agent and the stimulus. In natural circumstances, it is interpreted as 'the closer one gets to look at an object, the more details will be seen', and 'getting closer' needs motor effort. In our paradigm, the position and color 
112 of each dot were fixed within the trial, but in each frame (frame rate $60 \mathrm{fps}$ ) a different set of

113 randomly selected dots were made invisible so that the dots were 'blinking' with varying

114 phase and rate. The number of invisible dots in each frame was in proportion to the current

115 distance between the mouse cursor and each dot stimulus, thus the closer the cursor was to the

116 stimulus, the more dots were visible in a certain period (Fig 1B). When the cursor was moved

117 to the leftmost, the left group of dots would become completely visible and static, while the

118 right group would be completely invisible. Therefore, to get better sampling quality, subjects

119 must make some motor effort to move the cursor closer to the stimulus they want to examine.

121 Fig 1. Illustration of the paradigm. (A) A fixation $(1000-1500 \mathrm{~ms})$ on the start position with the mouse cursor was necessary to trigger each trial. After that, subjects moved the cursor to the stimuli alternatively to sample them. Finally, subjects clicked on the corresponding button to report which stimulus contained more white dots. (B) The number of invisible dots per frame was in proportion to the distance between the cursor and the stimulus. Subjects must move the cursor close to the stimulus to get better sampling quality. $80 \%$ range between the boundaries of the two stimuli, marked by a small white square on the screen. Subjects should drag the computer mouse cursor onto the square and stay fixed for a short time (1000 - $1500 \mathrm{~ms}$ randomly) to trigger the trial. After the fixation period, the two stimuli would appear, and the subject could start to sample them. Subjects were told to avoid pausing the cursor in the middle of the screen while looking sideways at the stimuli. We set

134 two sampling modes: In the one-switch mode, subjects should and could only make one 135 switching movement between the stimuli, which means they had only one chance to sample each of the alternatives. When the cursor was moved close enough to the stimulus (visible 
137 dots more than $90 \%$ per frame) and then left, that stimulus would be masked and could not be

138 examined again in the current trial. Subjects were instructed not to move their mouse to an 139 already masked stimulus. The length of time to examine each stimulus was not limited. In the

140 unlimited sampling mode, subjects could make as many switches and check each stimulus for

141 as many times as they needed.

142 The motor effort during the action stage took the form of moving the cursor to the 143 corresponding choice button and clicking on it to report the final choice. The choice buttons 144 were two small white squares displayed on the lower half of the screen, vertically $7 \mathrm{~cm}$ from 145 the centers of the stimuli. We set two types of trials differentiated by the location of the 146 choice buttons: In the first type, the buttons were horizontally centered, so the motor effort 147 (measured by the moving distance) to drag the cursor from the two stimuli to the buttons was 148 approximately the same. In the second type, the buttons were placed under the right stimulus, 149 so that the required motor effort would be less if the subject sampled the right stimulus last 150 and started from there to reach for the buttons.

151 The display screen size was $28.5 \times 18 \mathrm{~cm}$, resolution $1280 \times 800$ pixels, refresh rate $15260 \mathrm{~Hz}$. The screen was placed $50-70 \mathrm{~cm}$ in front of the subjects. System mouse acceleration 153 was disabled to make the cursor movement on the screen linearly map the actual movement of 154 the mouse. Subjects were told not to pick up the mouse from the surface of the desk amid 155 each trial. Mouse trajectory was recorded from the moment the trial was triggered to when a 156 button was clicked (sampling rate $60 \mathrm{~Hz}$ ). We also recorded the final decision in each trial.

157 The stimuli and mouse tracking codes were programmed in MATLAB Psychtoolbox-3. 
A total of 24 subjects participated in the study (13 females, age $20-30)$; all of them

were university students. Subjects wore glasses for vision correction if needed. The research

162

was approved by the institutional ethics committee of Eotvos Lorand University, Hungary.

163

All subjects provided informed written consent, and none declared any history of neurological

164

diseases.

To avoid previous experimental processes interfering with later sampling patterns, we divided the subjects into 3 groups, each containing 8 subjects, and each group of subjects only performed in a single experimental condition (Table 1). After 10 practice trials to get familiar with the paradigm, each subject performed 2 blocks of 60 trials. A short break $(5-10$ minutes) took place between the blocks. The complete experiment took approximately $40-$ 60 minutes per subject.

Table 1. Details of the experimental condition settings.

\begin{tabular}{ccccc}
\hline Condition & Sampling Mode & Choice Button Position & Female Subjects & Mean Age \\
\hline Control & unlimited switches & horizontally centered & $4 / 8$ & 25.9 \\
Right-Biased & unlimited switches & under the right stimulus & $5 / 8$ & 26.1 \\
One-Switch & one switch only & horizontally centered & $4 / 8$ & 24.9 \\
\hline
\end{tabular}

172

173 Data analysis

\section{Sampling patterns}

Decision time for a trial was defined as the elapsed time from the onset of the stimuli

177 line between the sampling stage and the action stage was the moment when a downward y178 axis component of the cursor velocity exceeded the threshold. 
To test the linear relationship between the variables depicting sampling patterns, we

182 performed linear mixed-effects regressions with random effects for subject-specific intercepts and slopes.

\section{Psychometric curves}

Psychometric curves were fitted to the data pooled across all subjects within each

group or all simulation trials in the same condition using the generalized linear model (GLM)

187

with the logit link function.

188

\section{Comparing lines and curves}

slopes and intercepts:

$$
\boldsymbol{Y}=\beta_{0}+\beta_{1} \boldsymbol{X}+I\left(\beta_{2}+\beta_{3} \boldsymbol{X}\right)
$$

192

193

194

195

197

198

199

where $\beta_{0}, \beta_{1}, \beta_{2}$ and $\beta_{3}$ were free parameters, $\boldsymbol{X}$ was the predictor variable, and $I$ was the indicator variable whose value was 0 for the reference group and 1 for the other group.

To compare two psychometric curves, we fitted the data to the following logistic function:

$$
\boldsymbol{Y}=\frac{1}{1+e^{-\left(\beta_{0}+\beta_{1} X+I\left(\beta_{2}+\beta_{3} X\right)\right)}}
$$

Then, we tested the null hypotheses $\beta_{2}=0$ and $\beta_{3}=0$ with the two-tailed one-sample t-test to compare the intercepts and slopes (steepness) of the two curves.

\section{Bayesian Network modeling}


We listed all three possible Bayesian Network models for different hypotheses

202

203

204

205

206

207

208

209

210

211

212

213

214

215

216

217

218

219

220

221

222 regarding the causal relationship between the decision variable before the last sampling, the

last sampled item and the final choice in the trial. The conditional probability of choosing the right item given that it is last sampled was calculated under each hypothesis and compared with the empirical results.

For mathematical details of the models, see S1 Supporting Information.

To calculate the conditional probability $p$ (right chosen $\mid$ right last sampled) from the behavioral data, we first fitted a psychometric choice curve (probability of choosing the right item vs. difference between the proportions of white dots in the stimuli) to the trials in which the right item was sampled last for each subject individually, and then marginalized the difference between the stimuli. The mean $p$ (right chosen $\mid$ right last sampled) across the subjects in each group was compared with the value 0.5 (the probability without bias) using the one-tailed one-sample t-test. The One-Switch group and the Right-Biased group were compared with the Control group using Dunnett's test after a one-way ANOVA.

\section{aDDM simulation}

We built our aDDM following Krajbich et al. [15]. We set the relative value ( $r_{\text {left }}$ and $\left.r_{\text {right }}\right)$ to the proportion of white dots in each stimulus. The range of $r_{\text {left }}$ and $r_{\text {right }}$ in the experiment was $[0.25,0.75]$. The decision variable $(D V)$ started from 0 in each simulation trial, and the decision barriers were -1 for the left stimulus and +1 for the right stimulus. We applied the multiplicative model [22]. The drift rates $(v)$ in the model were defined as:

$$
\left\{\begin{array}{c}
v=d\left(r_{\text {left }}-\theta r_{\text {right }}\right), \text { left attended } \\
v=d\left(\theta r_{\text {left }}-r_{\text {right }}\right), \text { right attended }
\end{array}\right.
$$


223 where $d$ was the value scaling parameter, and $\theta$ was the multiplicative attentional discounting

224 parameter. Specifically, during the first sampling, the unattended stimulus was assigned a

225 mean value $r_{\text {mean }}=0.5$ instead of the real value because the subject had not sampled that

226 stimulus yet. Let $D V_{t}$ denote the value of the decision variable at time $t$. For every time step

$227 \Delta t$,

$$
D V_{t+\Delta t}=D V_{t}+v \Delta t+\varepsilon_{t}
$$

229

230

231

232

233

234

235

236

237

238

where $\varepsilon_{t}$ was drawn from a zero-mean Gaussian distribution with standard deviation $\sigma$. We assumed that the first sampling falls on the left stimulus with a fixed probability (Control: 0.59, One-Switch: 0.57, from empirical data), its duration drawn from a fixed gamma distribution. Each successive sampling epoch fell alternatively on the left and right stimulus and would continue until it reached a max time limit drawn from another fixed gamma distribution or until the decision variable reached one barrier. The parameters of the two gamma distributions were fitted with maximum likelihood estimation (MLE) to the empirical sampling time data in the Control condition. Time step $\Delta t$ was set to $10 \mathrm{~ms}$. For human subjects in the Control group, the max number of switches in a single trial was 10 , so we discarded simulations with more than 10 switches.

We fitted the three parameters in the model $(\theta, d$ and $\sigma)$ to the empirical data pooled across all subjects: For each set of parameters, we ran a fixed number of valid simulations (240 for the coarse search and 960 for the finer search) and compared the results with behavioral data using the following error metric:

$$
\operatorname{Err}=\left(\frac{y_{a}{ }^{\prime}-y_{a}}{y_{a}}\right)^{2}+\left(\frac{y_{n}{ }^{\prime}-y_{n}}{y_{n}}\right)^{2}
$$

where $y_{a}=0.9042$ and $y_{n}=2.0698$ were the accuracy and the mean number of switches calculated from the 960 trials pooled across the 8 subjects in the Control group, while $y_{a}{ }^{\prime}$ and 
$246 y_{n}{ }^{\prime}$ were the accuracy and the mean number of switches across all simulations. We performed

247 a grid search for the best fitting parameters: In the $i$-th iteration, we tested the parameter sets

248 given by the cross product of $\left\{\theta_{i 1}, \theta_{i 2}, \theta_{i 3}\right\},\left\{d_{i 1}, d_{i 2}, d_{i 3}\right\}$ and $\left\{\sigma_{i 1}, \sigma_{i 2}, \sigma_{i 3}\right\}$. Let $\left(\theta_{i}, d_{i}, \sigma_{i}\right)$

249 denote the parameters that generated the smallest Err value, then in the (i+1)-th iteration we

250 tested a finer grid given by the cross product of $\left\{\theta_{i}-0.5 \Delta \theta_{i}, \theta_{i}, \theta_{i}+0.5 \Delta \theta_{i}\right\},\left\{d_{i}-0.5 \Delta d_{i}, d_{i}\right.$,

$\left.251 d_{i}+0.5 \Delta d_{i}\right\}$ and $\left\{\sigma_{i}-0.5 \Delta \sigma_{i}, \sigma_{i}, \sigma_{i}+0.5 \Delta \sigma_{i}\right\}$, where $\Delta \theta_{i}, \Delta d_{i}$ and $\Delta \sigma_{i}$ were the step sizes used

252 in the $i$-th iteration. The initial values were $\{0.1,0.5,0.9\}$ for $\theta,\{0.001,0.005,0.009\}$ for $d$,

253 and $\{0.01,0.05,0.09\}$ for $\sigma$ in the coarse search and $\{0.6,0.7,0.8\}$ for $\theta,\{0.004,0.005,0.006\}$

254 for $d$, and $\{0.03,0.04,0.05\}$ for $\sigma$ in the finer search. We stopped the iterations when the step

255 sizes became smaller than $0.5 \%$ of the parameter values. The final fitting results were $\theta=$

$2560.67, d=0.0051$ and $\sigma=0.038$.

257 For the One-Switch condition, we used the same set of parameters $(\theta, d$ and $\sigma)$ in the

258 Control condition, but only two sampling epochs were allowed. The second sampling would

259 continue until the decision variable reached one barrier. We discarded the simulations in

260 which the second sampling exceeded $3000 \mathrm{~ms}$, which was the max duration of the second

261 sampling for $99.5 \%$ empirical trials in the One-Switch condition. The decision time for the

262 simulations was calculated by adding the mean transition time (delay between the sampling

263 epochs) measured from behavioral data to the total time length of the sampling epochs in the

264 simulations.

265 We simulated the model for 960 valid trials (the same sample size as the empirical 266 data pooled across all subjects in each condition) using the best fitting parameters above for

267 the Control condition and the One-Switch condition separately and compared the results with 268 human behavioral data. 


\section{Results}

\section{General sampling patterns}

272

273

274

275

276

277

278

279

280

281

282

283

284

285

286

Firstly, we studied the general sampling patterns in the Control condition. We plotted the horizontal mouse cursor position recorded during the sampling stage against elapsed time in each trial. Fig 2 shows the time series of the cursor position from a single block performed by one subject: The 60 trials in the block were sorted by the start position. The horizontal positions between the two stimuli were linearly mapped to $[0,1]$ and shown in a red-blue color scale. The typical sampling pattern was to switch the cursor once or multiple times between the two stimuli. The cursor paused mostly at either the leftmost or the rightmost, meaning that only one of the stimulus was clearly visible at a time. Therefore, we can assume that the eye gaze and the attention of the subject switched between the stimuli together with the cursor, which enables the comparison between our paradigm and former sequential sampling tasks and models.

Fig 2. Typical time series of the horizontal mouse cursor position during sampling. Data were from a single block (60 trials) performed by one subject in the Control group and sorted by the start position in each trial. Red color indicates that the current cursor position is closer to the right stimulus, while blue indicates that the cursor is closer to the left.

If a subject made $n$ switches in a trial, there would be $n+1$ sampling epochs alternatively assigned to the two stimuli. Assuming that each sampling period has approximately the same duration, the decision time should linearly correlate with the number of switches in each trial. Moreover, most of the motor effort during sampling was spent on 
293 switching the cursor from one stimulus to the other, the distance between them fixed.

294 Therefore, the total motor effort within a trial (measured by the horizontal moving distance of

295 the cursor) should also linearly correlate with the number of switches. Fig 3A shows the

296 histogram of the number of switches made in all 960 trials performed by subjects in the

297 Control group: In $42.5 \%$ trials only one switch was made, and the percentage of the trials

298 decreased as the switches made in them increased. Fig 3B and 3C show that the decision time

299 (linear mixed-effects regression: slope $=1276.6 \mathrm{~ms}, P=2.8 \times 10^{-21}$; Pearson's $r=0.78$ ) and

300 the horizontal moving distance (linear mixed-effects regression: slope $=17.8 \mathrm{~cm}, P=2.6 \times 10^{-}$

$301{ }^{54}$; Pearson's $r=0.90$ ) linearly correlated with the number of switches.

302

303

304

305

306

307

308

309

310

\section{1}

313

314

315

Fig 3. Number of switches within each trial, and its correlation with decision time and horizontal moving distance. (A) Histogram of the number of switches. (B) Linear correlation between decision time and number of switches during the trials. Mixed-effects regression: slope $=1276.6 \mathrm{~ms}, P=2.8 \times 10^{-21}$; Pearson's $r=0.78$. (C) Linear correlation between horizontal moving distance and number of switches during the trials. Mixed-effects regression: slope $=17.8 \mathrm{~cm}, P=2.6 \times 10^{-54}$; Pearson's $r=0.90$. Data included 960 trials pooled across 8 subjects in the Control group.

\section{Influences of motor effort on sampling patterns}

The total motor effort within one single trial consisted of three parts: first, to drag the cursor from the start position to the first sampled item; second, to switch between the items one or more times during sampling (each switch took approximately the same moving distance, as discussed previously); third and lastly, to drag the cursor from the last sampled 
316 item to the choice buttons. We studied how motor cost in the different parts interacted with

317 the decision-making process (Fig 4):

319 Fig 4. Right-Biased condition vs. Control condition. (A) Psychometric choice curves. (B)

320 Number of switches against trial difficulty, as measured by the absolute difference between

321 the proportions of white dots in the stimuli. (C) Psychometric curves for the first sampled

322 item. (D) Psychometric curves for the last sampled item. Error bars show 95\% confidence

323 intervals. Data included 960 trials pooled across 8 subjects in each condition.

Fig 4A shows the psychometric choice curves in the Control and the Right-Biased

conditions: There was no statistically significant difference between the two curves (intercept:

$P=0.0958$; slope: $P=0.1358$ ), and the overall accuracy was also similar (Control: $90.4 \%$;

328

Right-Biased: 91.5\%; unpaired two-tail t-test between individual subjects in the two groups: $P$

$=0.4599)$. The difference in motor costs during the action phase did not bias the decisions of

the subjects in our experimental paradigm. One possible reason is that the difference was not

directly related to the final choice; another possibility is that explicit knowledge of the motor cost would help to avoid integrating irrelevant factors into the decision to maintain high accuracy [23].

We plotted the number of switches made in the trials against trial difficulty (measured by the absolute difference between the proportions of white dots in the stimuli) in Fig 4B: In both conditions, the number of switches decreased with trial difficulty (significant slopes in

337 mixed-effects regression: $P=4.8 \times 10^{-10}$ for Control and $P=1.9 \times 10^{-8}$ for Right-Biased). There 338 was no significant difference between the two conditions (intercept: $P=0.5216$; slope: $P=$ 339 0.8516). The motor cost during sampling correlated with the number of switches, therefore we 
340 concluded that the more difficult the trials were, the more motor effort would be invested into

341 the sampling process.

342 Next, we examined the influences of motor cost upon the first and the last sampled

343 items. Fig 4C shows that the start position and the choice button position both affected the

344 first sampled item: In the Control condition, subjects tended to sample the item closer to the

345 start position, which would reduce the first part of the total motor cost. However, we observed

346 a systematic bias to sample the left item first, which may be related to the cultural habit of

347 dealing with items in left-to-right order (for example, people usually read from left to right).

348 In the Right-Biased condition, subjects showed an extra tendency to go for the left item first

349 (significantly different intercepts: $P=2.9 \times 10^{-9}$ ). The subject was most likely to make only

350 one switch (Fig 3A); in that case, starting from the left item would lead to taking the shorter

351 path from the right item to the buttons, reducing the last part of the motor effort. In Fig 4D we

352 plotted the probability of sampling the right item last against the difference between

353 proportions of white dots in the two stimuli: Generally, subjects were more likely to sample

354 the stimulus with more white dots last. In the Right-Biased condition, subjects preferred to

355 sample the right stimulus last (significantly different intercepts: $P=2.9 \times 10^{-6}$ ), which would

356 reduce the motor cost during the action phase.

357

358 Influences of the decision variable on sampling patterns

The last-sampling bias is the phenomenon that subjects are more likely to choose the

360 last sampled stimulus. Such a bias has been reported in several human decision-making

361 studies of both preferential and perceptual decisions [15, 17]. However, the causal

362 relationship behind the last-sampling bias is not completely clear: Do subjects tend to choose

363 a stimulus because it is the last sampled one, or do they tend to sample the particular stimulus 
364 last because they already want to choose it, or both? According to the aDDM, the evidence

365 accumulation rate for the stimulus not being sampled is discounted, so the decision variable

366 will be more likely to reach the barrier at the last sampled side [15]. Otherwise, the aDDM

367 assumes that the current decision variable has no backward influence upon sampling patterns.

368 There is rare evidence supporting that the temporary decision has a causal effect on the

369 allocation of attention during sampling [19].

370 Bayesian Network modeling

371 To study the causal relationship between the last sampled item and the decision, we

372 built a Bayesian Network model quantifying the size of the last-sampling bias. Fig 5 displays

373 the graphical models for the networks: Naturally, the final decision depends on the decision

374 variable. In the One-Switch condition, the last sampled item is the alternative of the first

375 sampled one, which in turn depends on the motor cost measured by the distance from the start

376 position to the stimulus. In the Right-Biased condition, the last sampled item depends on the

377 motor cost in the action stage. Apart from the common dependency structures described

378 above, there are three possible models with different hypotheses on whether the last sampled

379 item depends on the decision variable and whether the final choice depends on the last 380 sampled item:

382 Fig 5. Causal relationship for the last-sampling bias. Graphical models for possible hypotheses regarding the conditional dependency relationship between the decision variable before the last sampling, the last sampled stimulus and the final choice. Arrows show exist generally but absent in the specific experimental condition. 


\section{Model (a)}

Like the aDDM, the first model assumes that the final decision depends on the last

390

391

392

393

394

397

398

399

400

401

402

403

404

405

406

407

408

409 sampled item, but the last sampled item is independent of the decision variable. Therefore, in the Control condition we have:

$$
\begin{aligned}
& p(\text { right chosen } \mid \text { right last sampled }) \\
& =\int p(\text { right chosen } \mid \boldsymbol{D} \boldsymbol{V} \text {, right last sampled }) p(\boldsymbol{D} \boldsymbol{V}) d \boldsymbol{D} \boldsymbol{V}
\end{aligned}
$$

where $\boldsymbol{D} \boldsymbol{V}$ is the value of the decision variable exactly before the last sampling epoch starts. $p$ (right chosen | right last sampled) measures the size of the last-sampling bias. The bias is due to the term $p$ (right chosen $\mid \boldsymbol{D} \boldsymbol{V}$, right last sampled), which can be regarded as a function of $\boldsymbol{D} \boldsymbol{V}$ : For each given value of $\boldsymbol{D} \boldsymbol{V}, p($ right chosen $\mid \boldsymbol{D} \boldsymbol{V}$, right last sampled $)>p($ right chosen $\mid$ $\boldsymbol{D} \boldsymbol{V})$. In the aDDM, $p$ (right chosen $\mid \boldsymbol{D} \boldsymbol{V}$, right last sampled) is the probability for the decision variable to drift to the right boundary at the end of the last sampling, decided by the aDDM parameters $(d, \sigma$ and $\theta)$ and the relative values for the two stimuli.

In the One-Switch condition, the dependency structure between $\boldsymbol{D V}$, the last sampled item and the final choice remains the same, but the following analysis explains why the size of the last-sampling bias will increase (see our aDDM simulation results): At the beginning of each trial, the decision variable is set to 0 ; as the sampling time elapses, more drift steps $(v \Delta t$ $\left.+\varepsilon_{t}\right)$ are added to the decision variable, so its variance increases. When only two sampling epochs are allowed, the elapsed time before the last sampling is shorter, thus $p(\boldsymbol{D} \boldsymbol{V})$ will have a narrower variance. In that case, the product of a biased $p$ (right chosen $\mid \boldsymbol{D} \boldsymbol{V}$, right last sampled) and $p(\boldsymbol{D} \boldsymbol{V})$ will be larger than that in the Control condition.

In the Right-Biased condition, the value of $p$ (right chosen | right last sampled) will not change because no term in Equation (6) depends on the motor cost to reach for the buttons. 
412 but the final decision is independent of the last sampled item. Therefore, in the Control 413 condition,

$$
p \text { (right chosen | right last sampled) }
$$

$$
=\int p(\text { right chosen } \mid \boldsymbol{D} \boldsymbol{V}) \frac{p(\text { right last sampled } \mid \boldsymbol{D} \boldsymbol{V})}{p(\text { right last sampled })} p(\boldsymbol{D} \boldsymbol{V}) d \boldsymbol{D} \boldsymbol{V}
$$

415 Under that hypothesis, the last-sampling bias is due to the term $p$ (right last sampled $\mid \boldsymbol{D} \boldsymbol{V}$ ),

416 which is also a function of $\boldsymbol{D} \boldsymbol{V}$ : When $\boldsymbol{D} \boldsymbol{V}>0, p$ (right last sampled $\mid \boldsymbol{D} \boldsymbol{V})>p$ (right last

417 sampled); when $\boldsymbol{D} \boldsymbol{V}<0, p$ (right last sampled $\mid \boldsymbol{D} \boldsymbol{V})<p$ (right last sampled). $p$ (right last sampled) will rise by the same additive amount. Compared with the Control condition, the term $p$ (right last sampled $\mid \boldsymbol{D} \boldsymbol{V}) p\left(\right.$ right last sampled ${ }^{-1}$ will become smaller when $\boldsymbol{D} \boldsymbol{V}>0$ and larger when $\boldsymbol{D} \boldsymbol{V}<0$, resulting in a decreased size of the last-sampling bias.

Model (c)

$p$ (right chosen | right last sampled)

$$
=\int p(\text { right chosen } \mid \boldsymbol{D} \boldsymbol{V} \text {, right last sampled }) \frac{p(\text { right last sampled } \mid \boldsymbol{D} \boldsymbol{V})}{p(\text { right last sampled })} p(\boldsymbol{D} \boldsymbol{V}) d \boldsymbol{D} \boldsymbol{V}
$$


430 In Equation (9), the total bias in $p$ (right chosen | right last sampled) has two sources: $p$ (right

431 chosen $\mid \boldsymbol{D} \boldsymbol{V}$, right last sampled) and $p$ (right last sampled $\mid \boldsymbol{D} \boldsymbol{V})$. The last sampled item is more

432 likely to be chosen, and the temporarily winning item is more likely to be sampled last.

433 Under such assumptions, the last-sampling bias should remain in the One-Switch 434 condition because the term $p$ (right chosen $\mid \boldsymbol{D V}$, right last sampled) is biased, but the size will 435 decrease because the term $p($ right last sampled $\mid \boldsymbol{D} \boldsymbol{V})$ now disappears.

In the Right-Biased condition, $p$ (right chosen | right last sampled) will also become

437 smaller similar to that in Model (b).

438

439

440

441

442

443

444

\section{Model predictions vs. empirical results}

Let $p_{\text {Control, }}, p_{\text {One-Switch }}$ and $p_{\text {Right-Biased }}$ denote $p$ (right chosen $\mid$ right last sampled) in each specific experimental condition. We summarized different model predictions and the empirical results in Table 2: Among the three hypotheses, only Model (c) correctly predicted the behavioral data. Therefore, we concluded that the causal relationship between sampling patterns and the decision is bidirectional.

Table 2. Summary of different model predictions about the last-sampling bias and the empirical results.

\begin{tabular}{|c|c|c|c|}
\hline Model & Predictions & \multirow{4}{*}{ 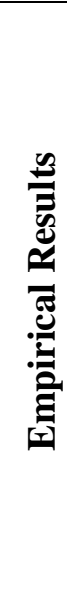 } & \multirow{4}{*}{$\begin{array}{c}p_{\text {Control }}>0.5^{* * *} \\
\text { (one-sample t-test, } P=2.0 \times 10^{-5} \text { ) } \\
p_{\text {One-Switch }}>0.5^{* *} \\
\text { (one-sample t-test, } P=0.0018 \text { ) } \\
p_{\text {One-Switch }}<p_{\text {Control }} * * * \\
\text { (Dunnett's test, } P=5.9 \times 10^{-4} \text { ) } \\
p_{\text {Right-Biased }}>0.5^{* *} \\
\text { (one-sample t-test, } P=0.0037 \text { ) } \\
p_{\text {Right-Biased }}<p_{\text {Control }} * * \\
\text { (Dunnett's test, } P=0.0018 \text { ) }\end{array}$} \\
\hline (a) & $\begin{array}{c}p_{\text {Control }}>0.5 \\
p_{\text {One-Switch }}>p_{\text {Control }}(\times) \\
p_{\text {Right-Biased }}=p_{\text {Control }}(\mathbf{x})\end{array}$ & & \\
\hline (b) & $\begin{array}{c}p_{\text {Control }}>0.5 \\
p_{\text {One-Switch }}=0.5(\times) \\
0.5<p_{\text {Right-Biased }}<p_{\text {Control }}\end{array}$ & & \\
\hline (c) & $\begin{array}{c}p_{\text {Control }}>0.5 \\
0.5<p_{\text {One-Switch }}<p_{\text {Control }} \\
0.5<p_{\text {Right-Biased }}<p_{\text {Control }}\end{array}$ & & \\
\hline
\end{tabular}


$p_{\text {Control}}, p_{\text {One-Switch }}$ and $p_{\text {Right-Biased }}$ denote $p$ (right chosen $\mid$ right last sampled) in each

449 experimental condition. The conditional probability measures the size of the last-sampling

450 bias, and a value of 0.5 means that there is no bias. $* * P<0.01$, ***P $<0.001$. The cross $(x)$

marks that the prediction contradicted empirical results.

\section{3 aDDM simulation}

On top of the theoretical Bayesian Network analysis, we also ran an aDDM simulation

to test whether the decision variable can feedback into the sampling patterns. In our

simulations, each sampling epoch was focused alternatively on the two stimuli until it reached

a time limit randomly drawn from a distribution fitted to the empirical data. In the One-

Switch condition, only one switch of attention was allowed. Each simulation ended when one

of the decision boundaries was reached. Therefore, the allocation of attention in the aDDM

was independent of the current decision variable. Fig 6 shows the comparison between simulated and empirical results:

Fig 6. aDDM simulation results vs. empirical data. (A) Psychometric choice curve in the

464 Control condition. (B) Number of switches in the Control group against trial difficulty, as

465 measured by the absolute difference between the proportions of white dots in the stimuli. (C)

466 Last-sampling bias in the Control group. The horizontal axis shows the difference between the

467 proportions of white dots between the last sampled stimulus and the other, while the vertical

468 axis shows the probability of choosing the last sampled stimulus. (D) Psychometric choice

469 curve in the One-Switch condition. (E) Decision time in the One-Switch group against trial

470 difficulty. (F) Last-sampling bias in the One-Switch group. Error bars show 95\% confidence 
471 intervals. Empirical data included 960 trials pooled across 8 subjects in each condition;

472 simulated data included 960 trials in each condition.

473

474 Firstly, we compared the psychometric choice curves: There was no statistically

475 significant difference between the psychometric choice curves for the simulations and the 476 human subjects (intercept: $P=0.0679$; slope: $P=0.0610$ ) in the Control condition (Fig 6A).

477 In the One-Switch condition, there was no significant difference between the intercepts of the 478 curves $(P=0.7116)$, but the slope (steepness) for the simulated data was significantly smaller 479 than that of the empirical data $\left(P=3.2 \times 10^{-4}\right)$, meaning that the overall accuracy in the 480 simulations was lower (Fig 6D). When only one switch was allowed, the choice accuracy of 481 the simulations reduced from $90.1 \%$ to $84.4 \%$, while for the human subjects there was no 482 significant reduction (unpaired one-tail t-test between individual subjects in the two groups: $P$ $483=0.8082)$

Next, we compared the number of switches in the Control condition (Fig 6B): There was no statistically significant difference between the simulated and empirical results regarding the number of switches against trial difficulty (intercept: $P=0.2690$; slope: $P=$ 0.2384). In the One-Switch condition, we compared the decision time instead of the number of switches (for it is constantly 1): There was no significant difference between the simulated and empirical results regarding the decision time against trial difficulty ((Fig 6E, intercept: $P$ $490=0.6125$; slope: $P=0.2257)$. probability of choosing the last sampled item against the difference between the proportions

493 of white dots between the last sampled stimulus and the other. All the curves had an intercept larger than 0.5 , showing a tendency to choose the last sampled item, but the sizes of the bias 
$496\left(P=5.8 \times 10^{-4}\right)$ in the Control condition and significantly higher than empirical $(P=0.0166)$ in

497 the One-Switch condition. Denying that subjects would switch back to sample the winning

498 item but assuming random switches all the while, the aDDM underestimated the last-sampling

499 bias in the Control condition and overestimated it in the One-Switch condition. The

500 simulation results matched our Bayesian Network analysis and implied that the current

501 decision had a causal effect on the sampling patterns.

502

503 Discussion

504 In summary, the adaptive sampling behavior during perceptual decision-making 505 exhibited the following patterns: First, the number of switches between the alternatives 506 correlated with the difficulty of the task: the more difficult the task was, the more times the

507 stimuli were resampled. Second, the sampling sequence was decided considering the start 508 position and the choice button position in an attempt to minimize the total motor effort. Third, 509 attention was biased to the eventually chosen item during the last sampling epoch. Combining 510 the modeling results, we concluded that both motor cost and the temporary decision have a causal influence upon the pattern of attention allocation during sampling.

Having reviewed recent computational models, behavioral studies and neural

513 recording results, Wispinski et al. [24] concluded that decision-making is a continuous

514 process from the presentation of behaviorally relevant options until movement completion.

515 Previous studies suggested that motor effort related to the action phase can influence the

516 decision [4-6, 8], and our results provided an extended conclusion that sampling behavior was

517 also influenced by the motor effort in different stages of the decision-making process. It 518 supported the idea that sensorimotor aspects should be considered as an actively integrated 519 part of the decision-making process. Further, several studies focused on the representation of 
520 motor effort and how it is related to cost minimization in decision-making as well as motor

521 control $[25,26]$; future studies may quantify the effect of motor cost on sampling behavior

522 with similar methods.

523

The relationship between attention and eye movement during decision-making has

524 been studied abundantly [27], but researches highlighting limb and body movements during

525 the sampling process are rare, even though in naturalistic circumstances such movements

526 usually cooperate with eye movements to sample relevant information better. In our research,

527 we designed a paradigm based on computer mouse tracking in which both gaze shift and hand

528 movement (moving the mouse) were necessary to switch attention between the options.

529 Although mouse tracking and eye tracking are both commonly applied process tracking

530 methods in decision-making research, their original purposes are slightly different: While eye

531 tracking mostly target on attention and information searching strategies, mouse cursor

532 tracking data reflect more about indecision and momentary preference [28]. In our paradigm,

533 however, subjects must move the cursor closer to get a better view of each stimulus, as if

534 approaching a real object to have a better look. In this way, the mouse trajectory can reflect

535 attention during sampling as eye traces did in previous studies. Moreover, our paradigm can

536 be applied to study eye-hand cooperation and coordination during decision-making as well.

538 subjects sample their options continuously until the relative evidence for one option reaches a

539 predetermined threshold, and such models capture the speed-accuracy trade-off phenomenon

540 well $[19,29,30]$. Interestingly, our results showed that subjects would make extra sampling

541 epochs during which the accuracy of the decision has not been improved significantly. One

542 possible explanation is that subjects were switching back to the previously sampled stimuli

543 again to verify their preliminary decision [31]. Similar to other studies [18, 32, 33], we

544 observed an attentional bias to the finally chosen option during the later sampling epochs. 
545 Mullett and Stewart [32] suggested that such a bias may be due to a relative instead of

546 absolute stopping rule. According to Krajbich [19], even as the decision variable evolves and

547 one option emerges as the winning one, it is still optimal to continue sampling information

548 randomly instead of favoring the leading option, since the information from both the winning

549 and losing options are of equal importance. However, the accuracy of the decision will not

550 necessarily decrease if the attentional bias happens at the later stage of sampling when the

551 main task is to validate the decision. This validating phase may be longer for perceptual

552 decisions, for people tend to respond with more caution in perceptual decisions than in

553 preferential decisions, especially when the stimuli are ambiguous [34]. Meanwhile, how the

554 sub-thresholds within the preliminary decision phase and the validating phase are determined

555 remains to be discussed.

556 Finally, our study provided evidence for the bidirectional causal relationship between

557 attention and decisions by Bayesian Network modeling. Bayesian Networks have been

558 customarily applied for probabilistic causal dependence assessment and inference in a wide

559 range of areas [35], including life science researches [36, 37]. It is capable of depicting and

560 predicting the conditional dependences between experimental variables through observed

561 data, thus becomes a beneficial tool for psychological studies. In our study, we listed all

562 possible network structures corresponding to different hypotheses on the causal relationship

563 between the last sampled item, the decision variable and the chosen item. Then, we compared

564 the predicted conditional probability of choosing the last sampled item with empirical data.

565 Contrary to previous literature [19], our results imply that rather than randomly switching

566 between the options, attention is drawn to the winning item during sampling. This finding

567 may lead to some modification to the basic assumptions of the aDDM in the future. 


\section{Acknowledgements}

570 We would like to express our sincere gratitude to Prof. László Mérő's supervision as

571 well as his support and encouragement. We are also grateful for the help on practical issues

572 from the Faculty of Education and Psychology ELTE and the kind participation of all the

573 subjects in the experiments.

574

575 References

576

577

578

579

580

581

582

583

584

585

586

587

588

589

590

591

592
1. Lepora NF, Pezzulo G. Embodied choice: how action influences perceptual decision making. PLoS computational biology. 2015 Apr 7;11(4):e1004110. doi: 10.1371/journal.pcbi.1004110

2. Connors BL, Rende R. Embodied Decision-Making Style: Below and Beyond Cognition. Frontiers in psychology. 2018;9. doi: 10.3389/fpsyg.2018.01123

3. Filimon F, Philiastides MG, Nelson JD, Kloosterman NA, Heekeren HR. How embodied is perceptual decision making? Evidence for separate processing of perceptual and motor decisions. Journal of Neuroscience. 2013 Jan 30;33(5):2121-36. doi: 10.1523/JNEUROSCI.2334-12.2013

4. Aczel B, Szollosi A, Palfi B, Szaszi B, Kieslich PJ. Is action execution part of the decision-making process? An investigation of the embodied choice hypothesis. Journal of Experimental Psychology Learning Memory and Cognition. 2018;44(6):918-926. doi: 10.1037/xlm0000484

5. Marcos E, Cos I, Girard B, Verschure PF. Motor cost influences perceptual decisions. PLoS One. 2015 Dec 16;10(12):e0144841. doi: 10.1371/journal.pone.0144841

6. Hagura N, Haggard P, Diedrichsen J. Perceptual decisions are biased by the cost to act. Elife. 2017 Feb 21;6:e18422. doi: 10.7554/eLife.18422 
593 7. de Lange FP, Fritsche M. Perceptual decision-making: picking the low-hanging fruit?.

594 Trends in cognitive sciences. 2017 May 1;21(5):306-7. doi: 10.1016/j.tics.2017.03.006

595 8. Burk D, Ingram JN, Franklin DW, Shadlen MN, Wolpert DM. Motor effort alters

596 changes of mind in sensorimotor decision making. PLoS One. 2014 Mar

597 20;9(3):e92681. doi: 10.1371/journal.pone.0092681

9. Harris CM, Wolpert DM. The main sequence of saccades optimizes speed-accuracy trade-off. Biological cybernetics. 2006 Jul 1;95(1):21-9. doi: 10.1007/s00422-006-

600 0064-

601

10. Armel KC, Beaumel A, Rangel A. Biasing simple choices by manipulating relative 602 visual attention. Judgment and Decision making. 2008;3(5):396-403.

603

11. Lim SL, O'Doherty JP, Rangel A. The decision value computations in the vmPFC and 604 striatum use a relative value code that is guided by visual attention. Journal of Neuroscience. 2011 Sep 14;31(37):13214-23. doi: 10.1523/JNEUROSCI.124611.2011

607

608

12. Atalay AS, Bodur HO, Rasolofoarison D. Shining in the center: Central gaze cascade effect on product choice. Journal of Consumer Research. 2012 May 3;39(4):848-66.

609 doi: $10.1086 / 665984$

610

13. Bird GD, Lauwereyns J, Crawford MT. The role of eye movements in decision making and the prospect of exposure effects. Vision Research. 2012 May 1;60:16-21. doi: 10.1016/j.visres.2012.02.014

614

14. Kunar MA, Watson DG, Tsetsos K, Chater N. The influence of attention on value integration. Attention, Perception, \& Psychophysics. 2017 Aug 1;79(6):1615-27. doi: 10.3758/s13414-017-1340-7 
616 15. Krajbich I, Armel C, Rangel A. Visual fixations and the computation and comparison

617 of value in simple choice. Nature neuroscience. 2010 Oct;13(10):1292. doi:

619

16. Krajbich I, Rangel A. Multialternative drift-diffusion model predicts the relationship between visual fixations and choice in value-based decisions. Proceedings of the National Academy of Sciences. 2011 Aug 16;108(33):13852-7. doi: 10.1073/pnas. 1101328108

17. Tavares G, Perona P, Rangel A. The attentional drift diffusion model of simple perceptual decision-making. Frontiers in neuroscience. 2017 Aug 24;11:468. doi: 10.3389/fnins.2017.00468 preference. Nature neuroscience. 2003 Dec;6(12):1317. doi: 10.1038/nn1150

19. Krajbich I. Accounting for attention in sequential sampling models of decision making. Current opinion in psychology. 2018 Oct 13. doi: 10.1016/j.copsyc.2018.10.008

20. Gottlieb J. Understanding active sampling strategies: Empirical approaches and implications for attention and decision research. Cortex. 2018 May 1;102:150-60. doi: 10.1016/j.cortex.2017.08.019

21. Ludwig CJ, Evens DR. Information foraging for perceptual decisions. Journal of Experimental Psychology: Human Perception and Performance. 2017 Feb;43(2):245. doi: $10.1037 / x h p 0000299$

22. Smith SM, Krajbich I. Gaze amplifies value in decision making. Psychological science. 2019 Jan;30(1):116-28. doi: 10.1177/0956797618810521

23. Hagura N, Diedrichsen J, Haggard P. Action cost biases the perceptual decision making, only when the cost is implicit. Translational and Computational Motor Control, 2013. 
641 24. Wispinski NJ, Gallivan JP, Chapman CS. Models, movements, and minds: bridging

642 the gap between decision making and action. Annals of the New York Academy of

643 Sciences. 2018 Oct 1. doi: 10.1111/nyas.13973

644 25. Shadmehr R, Huang HJ, Ahmed AA. A representation of effort in decision-making

645 and motor control. Current biology. 2016 Jul 25;26(14):1929-34. doi:

$646 \quad 10.1016 /$ j.cub.2016.05.065

647 26. Morel P, Ulbrich P, Gail A. What makes a reach movement effortful? Physical effort

648 discounting supports common minimization principles in decision making and motor

649 control. PLoS biology. 2017 Jun 6;15(6):e2001323. doi:

650

10.1371/journal.pbio.2001323

651

27. Orquin JL, Loose SM. Attention and choice: A review on eye movements in decision

652

653 making. Acta psychologica. 2013 Sep 1;144(1):190-206. doi:

654 10.1016/j.actpsy.2013.06.003

655

656

657

28. Schulte-Mecklenbeck M, Johnson JG, Böckenholt U, Goldstein DG, Russo JE, Sullivan NJ, Willemsen MC. Process-tracing methods in decision making: On growing up in the 70s. Current Directions in Psychological Science. 2017

659

660 Oct;26(5):442-50. doi: 10.1177/0963721417708229

29. Heitz RP. The speed-accuracy tradeoff: history, physiology, methodology, and

661 behavior. Frontiers in neuroscience. 2014 Jun 11;8:150. doi:

662 10.3389/fnins.2014.00150

30. Forstmann BU, Ratcliff R, Wagenmakers EJ. Sequential sampling models in cognitive neuroscience: Advantages, applications, and extensions. Annual review of psychology. 
664

665

666

667

668

669

670

671

672

673

674

675

676

677

678

679

680

681

682

683

684

685 Supporting information

686

687 10.1371/journal.pone.0078993 0941-1 $10.1109 / 3468.541341$

31. Cassey TC, Evens DR, Bogacz R, Marshall JA, Ludwig CJ. Adaptive sampling of information in perceptual decision-making. PloS one. 2013 Nov 27;8(11):e78993. doi:

32. Mullett TL, Stewart N. Implications of visual attention phenomena for models of preferential choice. Decision. 2016 Oct;3(4):231. doi: 10.1037/dec0000062

33. Onuma T, Penwannakul Y, Fuchimoto J, Sakai N. The effect of order of dwells on the first dwell gaze bias for eventually chosen items. PloS one. 2017 Jul 19;12(7):e0181641. doi: 10.1371/journal.pone.0181641

34. Dutilh G, Rieskamp J. Comparing perceptual and preferential decision making. Psychonomic bulletin \& review. 2016 Jun 1;23(3):723-37. doi: 10.3758/s13423-015-

35. Heckerman D, Breese JS. Causal independence for probability assessment and inference using Bayesian networks. IEEE Transactions on Systems, Man, and Cybernetics-Part A: Systems and Humans. 1996 Nov;26(6):826-31. doi:

36. Friedman N. Inferring cellular networks using probabilistic graphical models. Science. 2004 Feb 6;303(5659):799-805. doi: 10.1126/science.1094068

37. Yu J, Smith VA, Wang PP, Hartemink AJ, Jarvis ED. Advances to Bayesian network inference for generating causal networks from observational biological data. Bioinformatics. 2004 Jul 29;20(18):3594-603. doi: 10.1093/bioinformatics/bth448

S1 Supporting Information. Mathematical details of the Bayesian Network modeling. S2 Video. Demo trials recorded from the screen. 


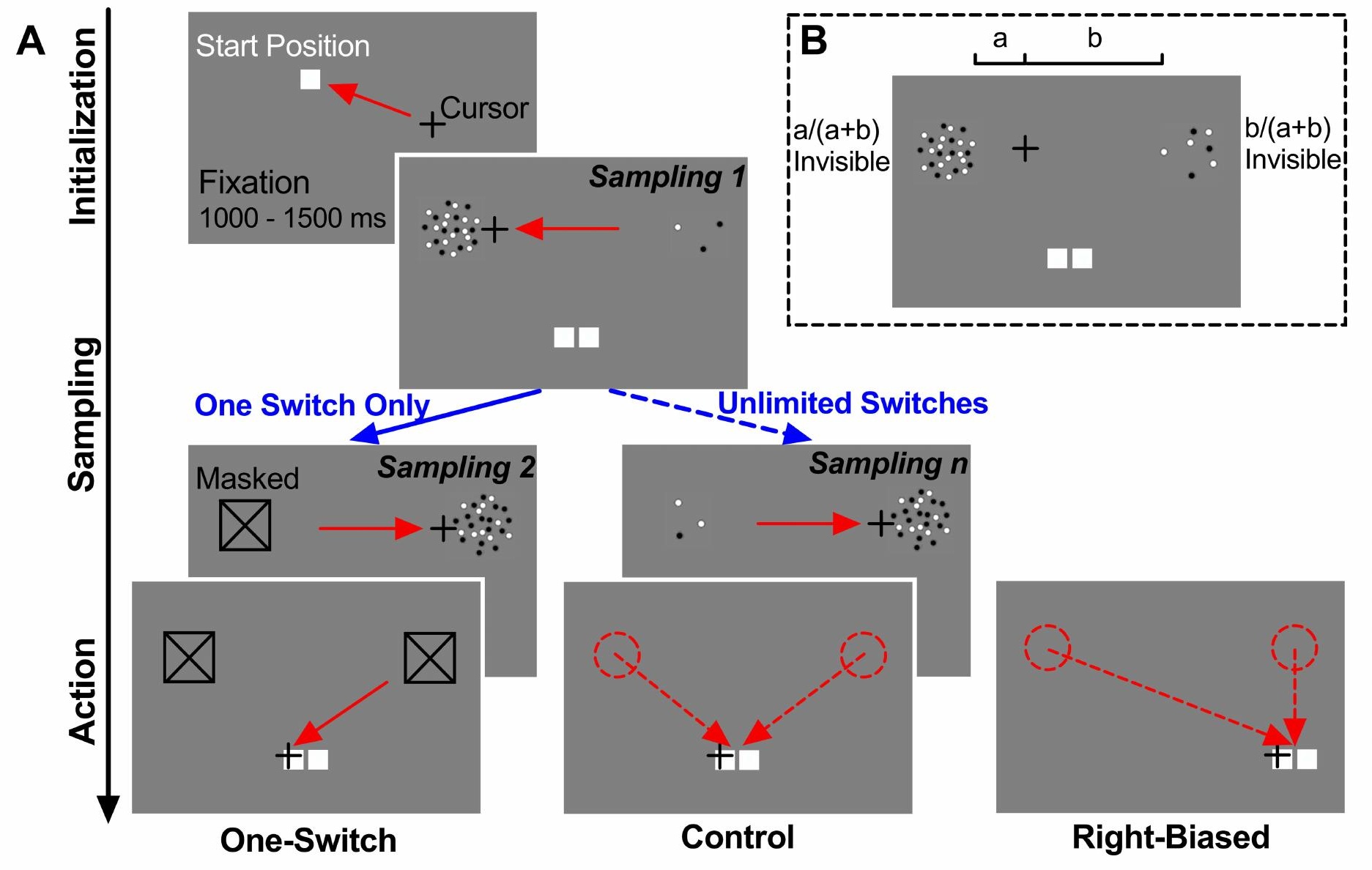




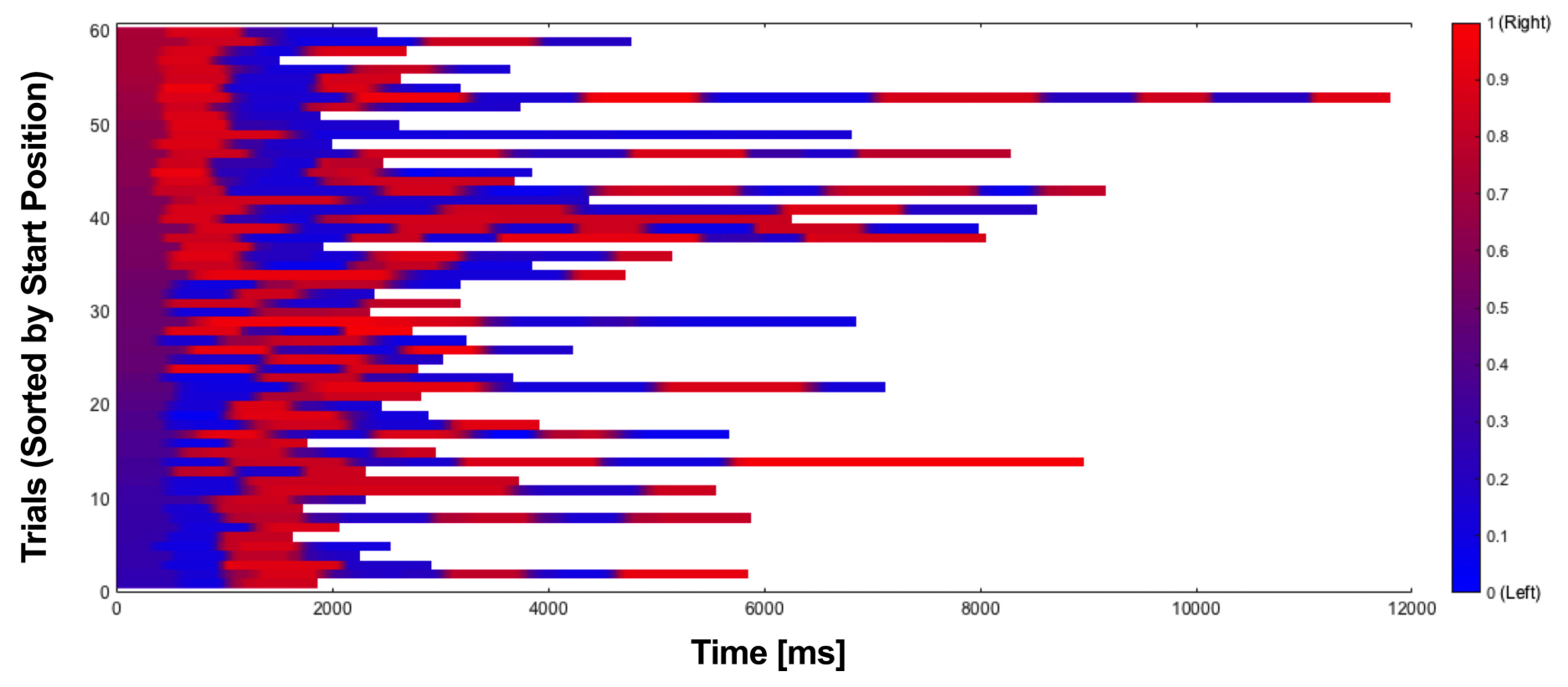




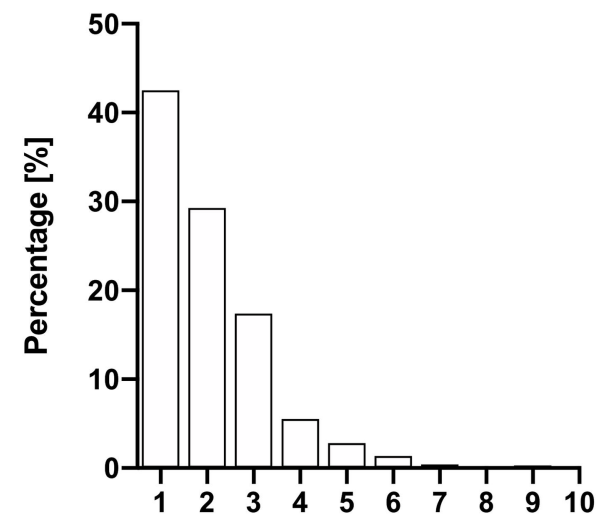

Number of Switches

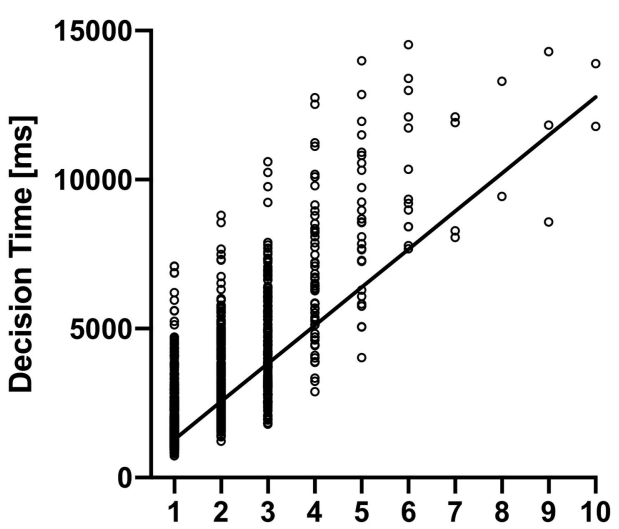

Number of Switches

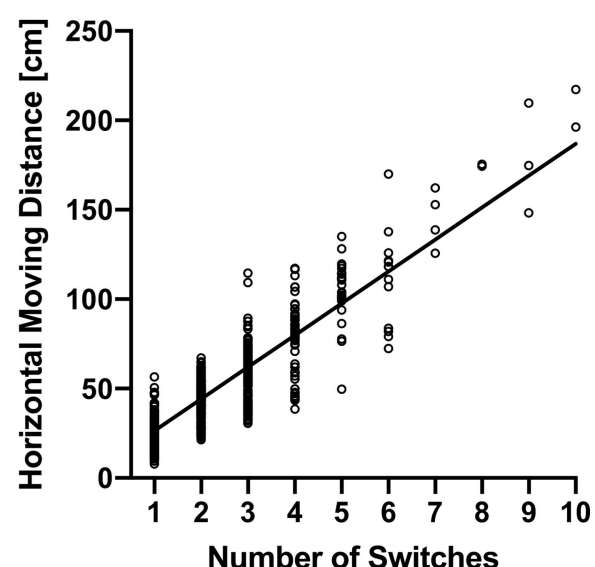

Number of Switches 

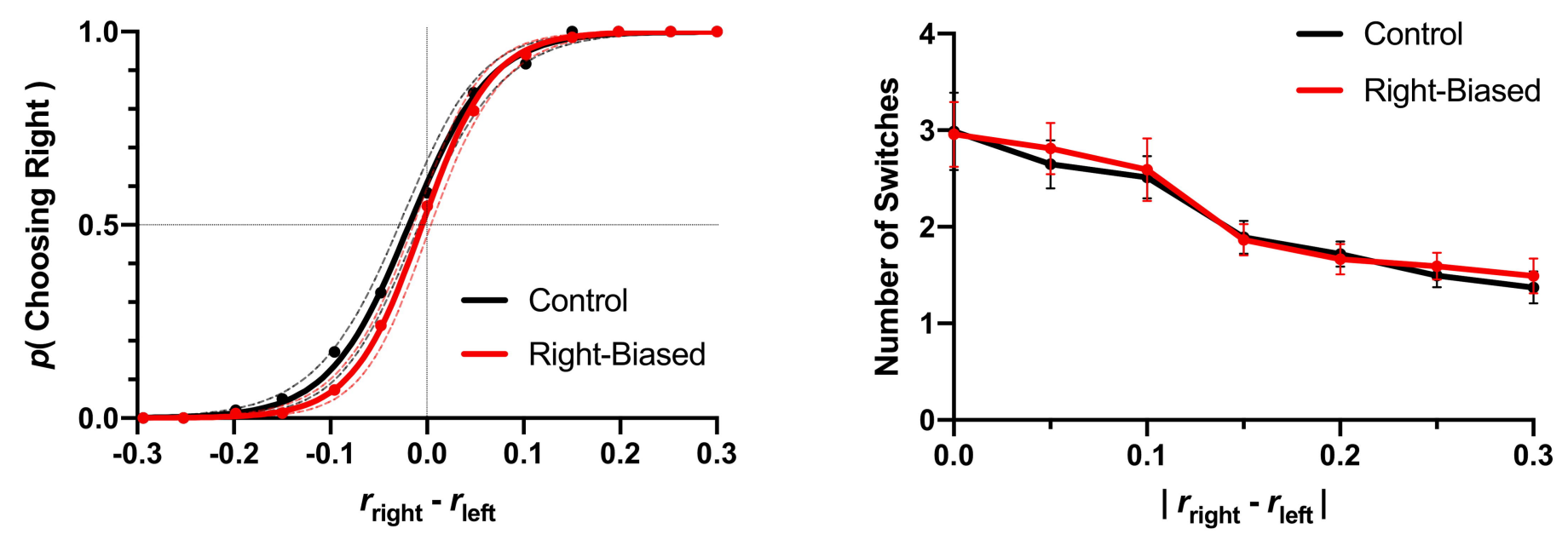

D
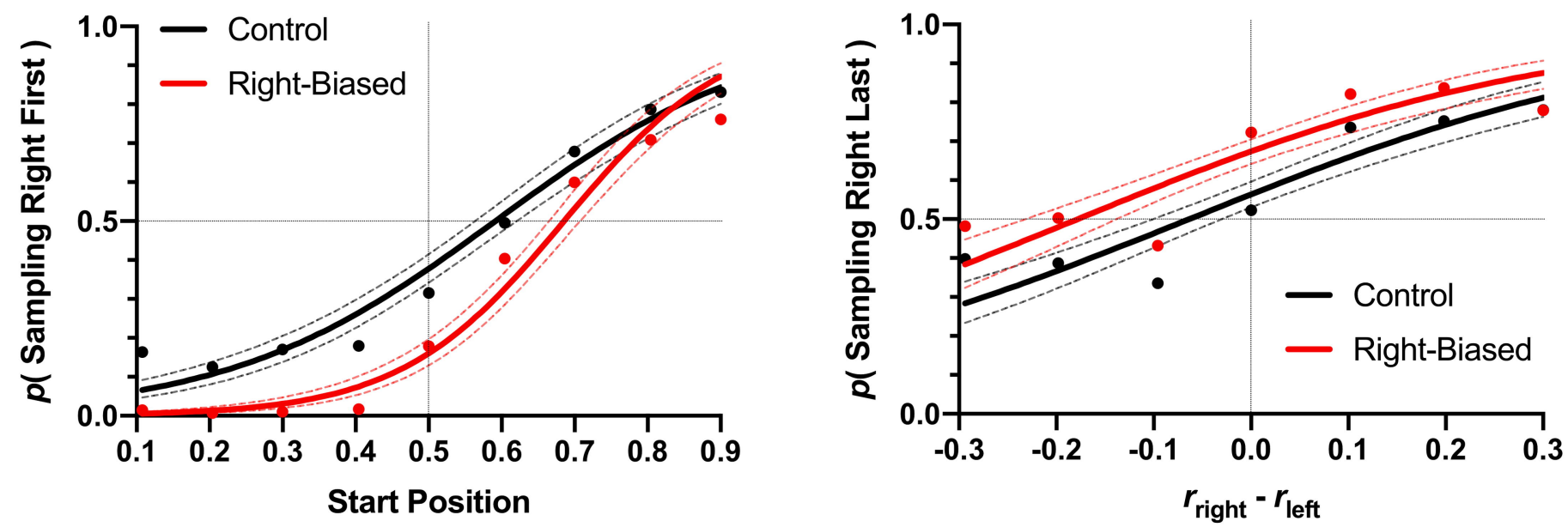


\section{Model (a)}

\section{Model (b)}
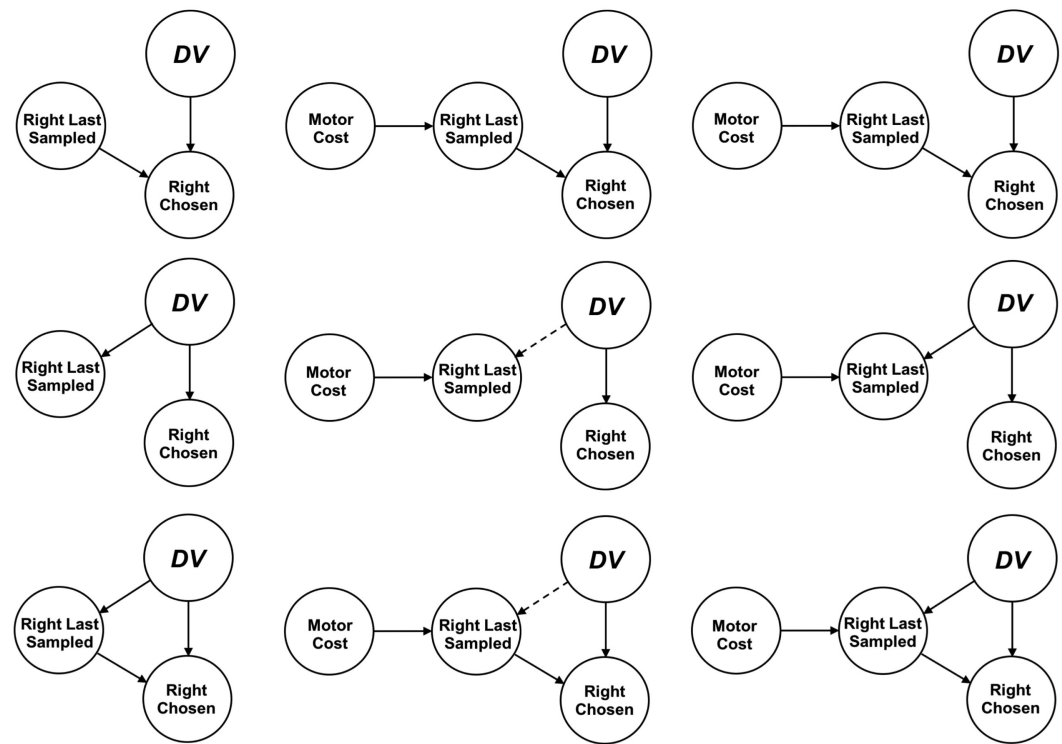
A $\quad$ Control

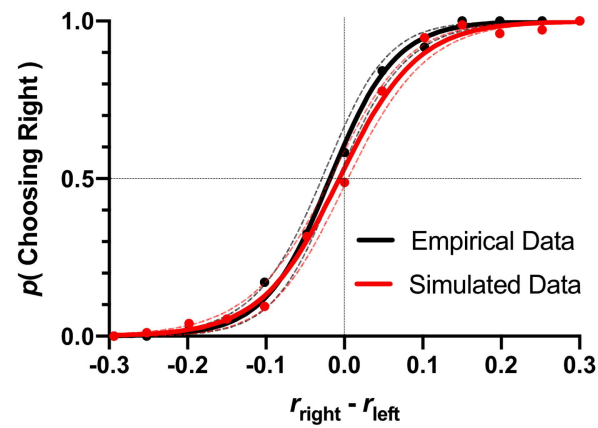

D

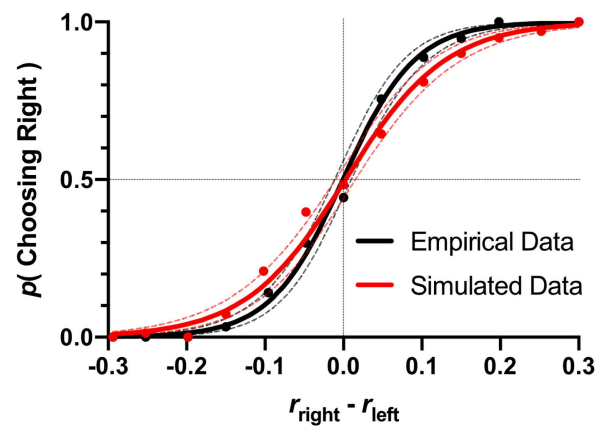

Control

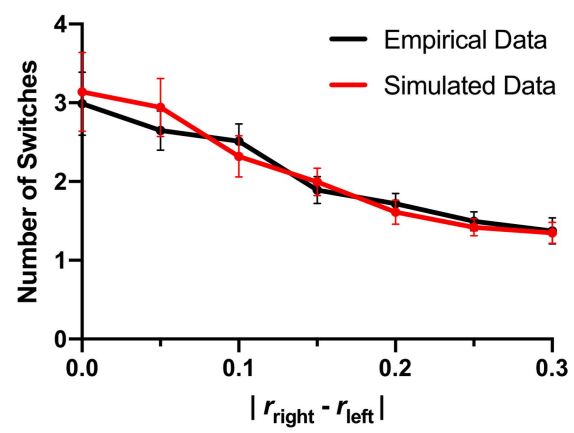

E

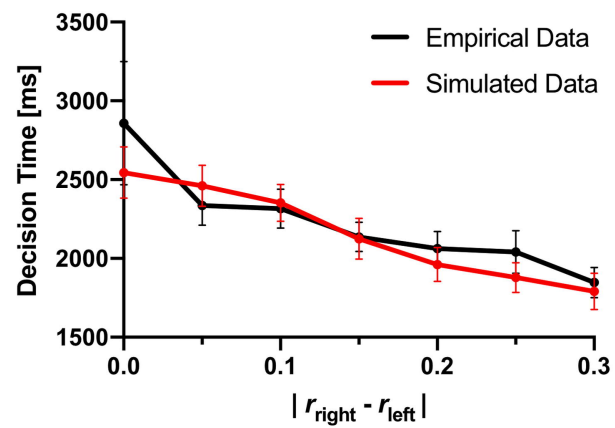

C

Control
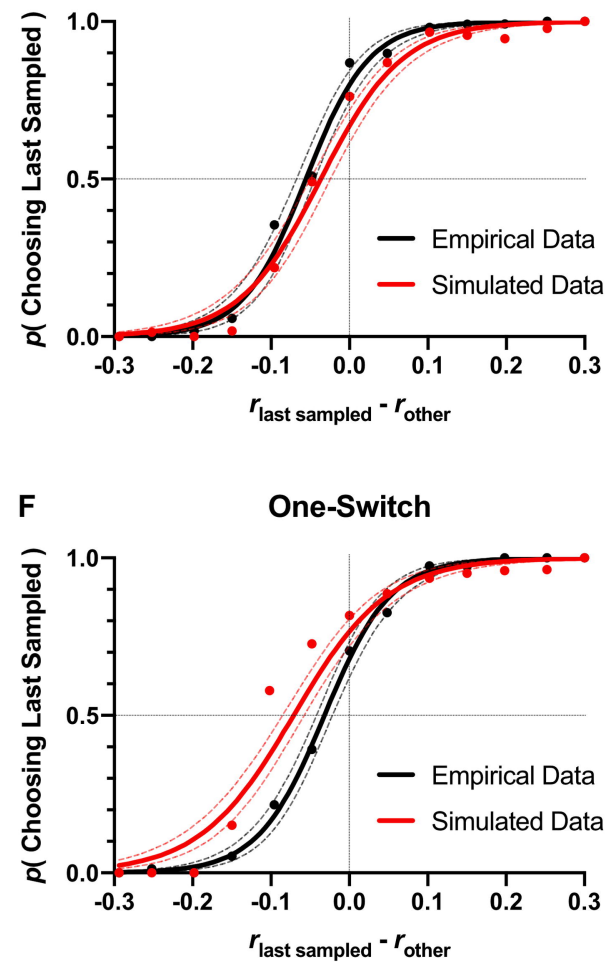